\title{
Geons with spin and charge
}

\author{
Jorma Louko ${ }^{1 *}$ Robert B. Mann ${ }^{2,3 \dagger}$ and Donald Marolf $4 \ddagger$ \\ ${ }^{1}$ School of Mathematical Sciences, University of Nottingham, Nottingham NGr 2RD, UK \\ ${ }^{2}$ Perimeter Institute for Theoretical Physics, Ontario N2J 2W9, Canada \\ ${ }^{3}$ Department of Physics, University of Waterloo Waterloo, Ontario N2L 3G1, Canada \\ ${ }^{4}$ Physics Department, UCSB, Santa Barbara, CA 93106, USA
}

(Revised February 2005)

$\langle\operatorname{arXiv:gr-qc/0412012\rangle }$

\begin{abstract}
We construct new geon-type black holes in $D \geq 4$ dimensions for Einstein's theory coupled to gauge fields. A static nondegenerate vacuum black hole has a geon quotient provided the spatial section admits a suitable discrete isometry, and an antisymmetric tensor field of rank 2 or $D-2$ with a pure $\boldsymbol{F}^{2}$ action can be included by an appropriate (and in most cases nontrivial) choice of the field strength bundle. We find rotating geons as quotients of the Myers-Perry(AdS) solution when $D$ is odd and not equal to 7 . For other $D$ we show that such rotating geons, if they exist at all, cannot be continuously deformed to zero angular momentum. With a negative cosmological constant, we construct geons with angular momenta on a torus at the infinity. As an example of a nonabelian gauge field, we show that the $D=4$ spherically symmetric $\mathrm{SU}(2)$ black hole admits a geon version with a trivial gauge bundle. Various generalisations, including both black-brane geons and Yang-Mills theories with Chern-Simons terms, are briefly discussed.
\end{abstract}

\footnotetext{
*jorma.louko@nottingham.ac.uk

†mann@avatar.uwaterloo.ca

${ }^{\ddagger}$ marolf@physics.ucsb.edu
} 


\section{Contents}

1 Introduction $\quad 2$

2 Static Einstein-U(1) geons $\quad 4$

2.1 Gibbons-Wiltshire metrid . . . . . . . . . . . . . . . . . . . . . . . 4

2.2 Geon quotient . . . . . . . . . . . . . . . . . . 6

2.3 Examples: Constant curvature transversal space . . . . . . . . . . . . . . 9

2.4 Generalisations . . . . . . . . . . . . . . . . . . 10

3 Angular momentum 11

3.1 Torus . . . . . . . . . . . . . . . . . . . . . . . . . . . . . . . . . . . . 13

3.2 Sphere . . . . . . . . . . . . . . . . . . . . . . . 13

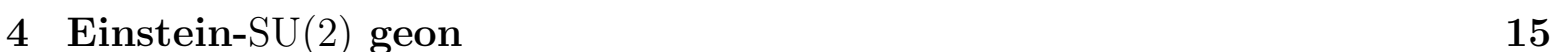

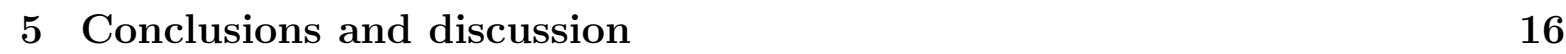

\section{Introduction}

The term "geon", short for "gravitational-electromagnetic entity", was introduced in 1955 by John Archibald Wheeler to denote a classical gravitational configuration, possibly coupled to electromagnetism or other zero-mass fields, that appears as a long-lived massive object when observed from a distance but is not a black hole [1, 2, 3, 4, 15, 6, While a precise definition of a geon seems not to have been sought, the examples studied in [1, 2, 3, 4, 5, 6] had spatial topology $\mathbb{R}^{3}$ and with one exception an asymptotically flat infinity, where mass could be defined by what are now known as ADM methods. The one exception was Melvin's magnetic universe [4, 7], which was subsequently deemed not to qualify as a geon, precisely because of its cylindrical infinity [8]. Unfortunately, while Melvin's universe is stable [8, 9, 10, these geons were not, owing to the tendency of a massless field either to disperse to infinity or to collapse into a black hole. This tendency has more recently been much studied in the context of critical phenomena in gravitational collapse [11.

In 1985, Sorkin [12 denoted by "topological geons" gravitational configurations whose spatial geometry is asymptotically flat and has the topology of a compact manifold with one puncture, the omitted point being at the asymptotic infinity. This generalises Wheeler's geon in the sense of allowing nontrivial spatial topology and a black hole horizon. An example of a topological geon that is a black hole is the space and time orientable $\mathbb{Z}_{2}$ quotient of the Kruskal manifold known as the $\mathbb{R P}^{3}$ geon [13. This spacetime has an asymptotically flat exterior region isometric to a standard Schwarzschild exterior and is hence an eternal black hole. However, while in Kruskal the spatial hypersurfaces are $\mathbb{R} \times S^{2}$ wormholes with two asymptopias, the spatial topology of the $\mathbb{R P}^{3}$ geon is 
$\mathbb{R} \mathbb{P}^{3} \backslash\{$ point at infinity $\} .^{1}$

As an eternal black and white hole spacetime, the $\mathbb{R P}^{3}$ geon is not an object one would expect to be formed in astrophysical processes. Its interest is that as an unconventional black hole, it provides an arena for probing our understanding of black holes both in the classical and quantum contexts. For example, in [13 the $\mathbb{R P}^{3}$ geon was used to illustrate a classical topological censorship theorem, and the Hamiltonian dynamics of spherically symmetric spacetimes with geon-type boundary conditions was investigated in [19, 20]. The Hawking(-Unruh) effect on the $\mathbb{R P}^{3}$ geon was analysed for scalar and spinor fields in 18, 21, addressing questions about the geon's entropy and its statistical mechanical interpretation. The $(2+1)$ dimensional asymptotically AdS analogue of the $\mathbb{R P}^{3}$ geon was used in 22, 23, 24] to probe AdS/CFT correspondence.

The purpose of this paper is to present new families of geon-type black holes in $D \geq 4$ spacetime dimensions for Einstein's theory coupled to gauge fields. We shall not attempt to give a precise definition of "geon-type black hole", but we require the spacetimes to be time orientable and foliated by spacelike hypersurfaces with a single asymptotic region. We also require the asymptotic region to be stationary and 'simple' in a sense that allows conserved charges to be defined by appropriate integrals. We also consider geon variants of certain black branes that appear in string theory. We find that while the $\mathbb{R P}^{3}$ geon generalises readily into static vacuum geons in any dimension, the inclusion of $\mathrm{U}(1)$ gauge fields is more subtle and depends sensitively on the dimension, in most cases requiring the field strength to be a section of a bundle that is twisted by the spatial fundamental group or by some quotient thereof. Such twisting is in particular required for electrically charged Reissner-Nordström in any spacetime dimension and for magnetically charged Reissner-Nordström in even spacetime dimensions. Similarly, while the rotating $(2+1)$-dimensional black hole has geon-type variants for any values of the angular momentum [25, 26, 27, we find that that the options for building geons from the rotating Kerr-Myers-Perry(-AdS) solutions [28, 29, 30, 31, 32, depend sensitively on the dimension. With a negative cosmological constant, we also present rotating geons with a flat horizon. As an example of a nonabelian gauge field, we show that the $D=4$ spherically symmetric $\mathrm{SU}(2)$ black hole [33, 34, 35, 36, 37, 38, 39] admits a geon variant with a trivial gauge bundle.

We use metric signature $(-++\cdots)$. Static Einstein-U(1) geons are discussed in section 2 and geons with angular momentum in section 3 . The $D=4$ spherically symmetric $\mathrm{SU}(2)$ geon is constructed in section 4. Section 5 presents a summary and concluding remarks.

\footnotetext{
${ }^{1}$ The time-symmetric initial data for the $\mathbb{R P}^{3}$ geon was discussed prior to [13] in [5] and [14, 15]. An early discussion on quotients of Kruskal can be found in [16 and a modern one in 17. The Euclidean-signature section of the $\mathbb{R}^{3}$ geon is discussed in [18.
} 


\section{Static Einstein-U(1) geons}

In this section we discuss Einstein-U(1) geons that have a static asymptotic region. In subsection 2.1 we recall relevant properties of the nondegenerate Gibbons-Wiltshire black holes with static asymptotic regions [40, and in subsection 2.2 we construct geon versions of these holes. Subsection 2.3 presents examples in the special case of constant curvature transversal space. Generalisations beyond the Gibbons-Wiltshire spacetimes, including generalisations to multiple (i.e., $[\mathrm{U}(1)]^{n}$ ) gauge fields, are discussed in subsection in 2.4

\subsection{Gibbons-Wiltshire metric}

Let $D \geq 4$, and let $\left(\overline{\mathcal{M}}, \overline{\mathrm{d} s^{2}}\right)$ be a $(D-2)$-dimensional positive definite Einstein manifold: Writing $\overline{\mathrm{d} s^{2}}=\bar{g}_{I J} \mathrm{~d} y^{I} \mathrm{~d} y^{J}$, we have $\bar{R}_{I J}=\bar{\lambda}(D-3) \bar{g}_{I J}$, where $\bar{\lambda} \in \mathbb{R}$ is a constant. In local Schwarzschild-like coordinates, the $D$-dimensional Lorentz-signature GibbonsWiltshire metric reads $40{ }^{2}$

$$
\begin{aligned}
\mathrm{d} s^{2} & =-\Delta \mathrm{d} t^{2}+\frac{\mathrm{d} r^{2}}{\Delta}+r^{2} \overline{\mathrm{d} s^{2}} \\
\Delta & =\bar{\lambda}-\frac{\mu}{r^{D-3}}+\frac{Q^{2}}{r^{2(D-3)}}-\frac{2 \Lambda r^{2}}{(D-1)(D-2)},
\end{aligned}
$$

where $r>0, \mu$ and $Q$ are real-valued constants and $\Lambda$ is the cosmological constant. The spacetime has a Maxwell field whose Faraday two-form is

$$
\boldsymbol{F}=\alpha_{D} \frac{Q}{r^{D-2}} \mathrm{~d} t \wedge \mathrm{d} r
$$

where $\alpha_{D}$ is a $D$-dependent constant whose precise value will not be needed in what follows. Equations (2.1) and (2.2) solve the Einstein-Maxwell equations, obtained from the action whose gravitational part is proportional to $\int \sqrt{-g}(R-2 \Lambda)$ and Maxwell part to $\int \sqrt{-g} F_{a b} F^{a b}$. When $\bar{\lambda}$ is nonzero, it can be normalised to $|\bar{\lambda}|=1$ without loss of generality by rescaling $r, t, \mu$ and $Q$.

On par with the 'electric' solution given by (2.1) and (2.2), we consider the 'magnetic' solution [55] in which (2.2) is replaced by

$$
\boldsymbol{H}=\alpha_{D}^{\prime} Q \boldsymbol{V} \overline{\mathrm{d} s^{2}}
$$

where $\alpha_{D}^{\prime}$ is a $D$-dependent constant and $\boldsymbol{V} \overline{\mathrm{d} s^{2}}$ is the volume form (respectively volume density) on $\left(\overline{\mathcal{M}}, \overline{\mathrm{d} s^{2}}\right)$ if $\overline{\mathcal{M}}$ is orientable (nonorientable) [56. Equations (2.1) and (2.3) solve the Einstein-U(1) equations obtained from the action whose gauge field part is proportional to $\int \sqrt{-g} H_{a \ldots f} H^{a \ldots f}$.

Henceforth we assume $\left(\overline{\mathcal{M}}, \overline{\mathrm{d} s^{2}}\right)$ to be geodesically complete.

\footnotetext{
${ }^{2}$ For related work, see 41, 42, 43, 44, 45, 46, 47, 48, 49, 50, 51, 52, 53, 54.
} 
We are interested in the situation in which the metric (2.1) extends into an eternal black-and-white hole spacetime in which a bifurcate event horizon at $\Delta=0$ [57] separates two exterior regions, both of which are static with respect to the timelike Killing vector $\partial_{t}$ and extend to a spacelike infinity. This can only occur for $\Lambda \leq 0$, as otherwise $\partial_{t}$ is not timelike at large $r$. When $\Lambda=0$, it is necessary that $\bar{\lambda}>0$, and the parameter range analysis and the conformal diagrams with suppressed $\left(\overline{\mathcal{M}}, \overline{\mathrm{d} s^{2}}\right)$ are as for Kruskal and nonextremal Reissner-Nordström [58]. When $\Lambda<0, \bar{\lambda}$ may take any value, and the parameter range analysis and the conformal diagrams are as in the four-dimensional case [51, 59, with the correction pointed out in [60, 61] to those diagrams that are shown in [51, 59] as squares. ${ }^{3}$

Let $\left(\mathcal{M}, \mathrm{d} s^{2}\right)$ denote the spacetime consisting of the four conformal blocks adjacent to the Killing horizon in the conformal diagram. We introduce in $\left(\mathcal{M}, \mathrm{d} s^{2}\right)$ standard global Kruskal null coordinates $(U, V, \boldsymbol{y})$, in which $\boldsymbol{y}$ denotes a point in $\overline{\mathcal{M}}$. The metric reads

$$
\mathrm{d} s^{2}=-f \mathrm{~d} U \mathrm{~d} V+r^{2} \overline{\mathrm{d} s^{2}},
$$

the horizon is at $U V=0$, and $f$ and $r$ are smooth positive functions of argument $U V$. The two-form (2.2) becomes

$$
\boldsymbol{F}=\alpha_{D} \frac{Q f}{2 r^{D-2}} \mathrm{~d} U \wedge \mathrm{d} V,
$$

while the expression (2.3) remains valid for $\boldsymbol{H}$. The static exteriors are at $U V<0$ and the interiors at $U V>0$. The range of $U V$ depends on the parameters; in particular, $U V$ is bounded below for $\Lambda<0$ but not for $\Lambda=0$. $\left(\mathcal{M}, \mathrm{d} s^{2}\right)$ may be extendible to the future and past through further Killing horizons, but whether or not it is, the conformal diagrams show that $\left(\mathcal{M}, \mathrm{d} s^{2}\right)$ contains all spacelike hypersurfaces that connect the two static regions and are given in the local metric (2.1) by a relation between $t$ and $r$. Working with $\left(\mathcal{M}, \mathrm{d} s^{2}\right)$ will thus suffice for our purposes. If $\left(\mathcal{M}, \mathrm{d} s^{2}\right)$ is extendible, the quotients in the rest of this paper can be extended in an obvious manner.

To summarise: The spacetime $\left(\mathcal{M}, \mathrm{d} s^{2}\right)$ is an eternal black hole, foliated by wormhole-like spacelike hypersurfaces of topology $\overline{\mathcal{M}} \times \mathbb{R}$. The static regions are locally asymptotically flat for $\Lambda=0$ and locally asymptotically anti-de Sitter for $\Lambda<0$. The topology at infinity, while possibly exotic, extends to the horizon and hence does not violate topological censorship theorems [13, 62, 63. When $\left(\overline{\mathcal{M}}, \overline{\mathrm{d} s^{2}}\right)$ is the round two-sphere and $Q=0=\Lambda,\left(\mathcal{M}, \mathrm{d} s^{2}\right)$ reduces to the Kruskal manifold.

The electric and magnetic solutions have respectively nonvanishing electric and magnetic charges at the infinities. Suppose first that $\overline{\mathcal{M}}$ is orientable, in which case $\left(\mathcal{M}, \mathrm{d} s^{2}\right)$ is both time and space orientable and the magnetic field strength (2.3) is a globallydefined $(D-2)$-form. In the magnetic solution, the magnetic charge density on a

\footnotetext{
${ }^{3}$ We exclude the case where the spacetime would be extendible past a coordinate singularity at $r=0$. Examination of $R_{a b c d} R^{a b c d}[52$ shows that this situation can only arise for $\mu=0=Q$, and it was seen in [51, 52] that this situation does then arise for some $\left(\overline{\mathcal{M}}, \overline{\mathrm{d} s^{2}}\right)$.
} 
hypersurface of constant $U$ and $V$ is the pull-back of $\boldsymbol{H}$, and (2.3) shows that the magnetic charge per unit volume of $\left(\overline{\mathcal{M}}, \overline{\mathrm{d} s^{2}}\right)$ is $\alpha^{\prime} Q$. In the electric solution, the electric charge density is defined by taking the Hodge dual of $\boldsymbol{F}$ and proceeding similarly, with the result that (with a suitable choice of orientation) the electric charge per unit volume of $\left(\overline{\mathcal{M}}, \overline{\mathrm{d} s^{2}}\right)$ is $\alpha Q$. From the globally-defined time and space orientations of $\left(\mathcal{M}, \mathrm{d} s^{2}\right)$ it follows that the charges at the two asymptopias have the opposite sign.

If $\overline{\mathcal{M}}$ is not orientable, these charge considerations generalise by first passing to the orientable double cover of $\overline{\mathcal{M}}$ and then taking the quotient. For Stokes' theorem on nonorientable manifolds in this context, see [64, 65, 66.

We have here presented the electric and magnetic solutions in terms of the field strengths, rather than in terms of gauge potentials. We shall do the same for the geon solutions in subsection 2.2. We shall return to the gauge potentials in section 5 .

\subsection{Geon quotient}

Suppose that $\left(\overline{\mathcal{M}}, \overline{\mathrm{d} s^{2}}\right)$ admits a freely-acting involutive isometry $P$, and let $\bar{\Gamma}:=$ $\left\{\operatorname{Id}_{\overline{\mathcal{M}}}, P\right\} \simeq \mathbb{Z}_{2}$ be the isometry group generated by $P$. The map

$$
J:(U, V, \boldsymbol{y}) \mapsto(V, U, P(\boldsymbol{y}))
$$

is then a freely-acting involutive isometry on $\left(\mathcal{M}, \mathrm{d} s^{2}\right)$, and it generates the isometry group $\Gamma:=\left\{\operatorname{Id}_{\mathcal{M}}, J\right\} \simeq \mathbb{Z}_{2} . J$ acts in the conformal diagram by the left-right reflection in the displayed two dimensions and by $P$ in the suppressed $D-2$ dimensions, from which it is seen that $J$ preserves the time orientation on $\left(\mathcal{M}, \mathrm{d} s^{2}\right)$ and maps the two static regions to each other. The case where the static regions are asymptotically locally flat and the nonstatic regions end at a spacelike singularity is shown in Figure 1.

The quotient $\left(\mathcal{M}_{g}, \mathrm{~d} s_{g}^{2}\right):=\left(\mathcal{M}, \mathrm{d} s^{2}\right) / \Gamma$ is a geon. For $Q=0,\left(\mathcal{M}_{g}, \mathrm{~d} s_{g}^{2}\right)$ clearly satisfies Einstein's equations. For $Q \neq 0$, there is a sublety in that the two-form (2.5) changes sign under $J$, and the $(D-2)$-density (2.3) may change sign under $J$ depending on the $P$-action on $\left(\overline{\mathcal{M}}, \overline{\mathrm{d} s^{2}}\right)$. Hence $\left(\mathcal{M}_{g}, \mathrm{~d} s_{g}^{2}\right)$ with the quotient gauge field solves the Einstein-U(1) field equations iff the field strength can be interpreted as a section of a bundle whose twisting is compatible with these sign changes. We now show that the field strength has this interpretation and describe the bundles in terms of $\pi_{1}\left(\mathcal{M}_{g}\right)$.

Let $\left(\widetilde{\mathcal{M}}, \widetilde{\mathrm{d} s^{2}}\right)$ be the universal covering space of $\left(\mathcal{M}, \mathrm{d} s^{2}\right)$, and let $\left(\widetilde{\mathcal{M}}, \widetilde{\mathrm{d} s^{2}}\right)$ be the universal covering space of $\left(\overline{\mathcal{M}}, \overline{\mathrm{d} s^{2}}\right)$. On $\left(\widetilde{\mathcal{M}}, \widetilde{\mathrm{d} s^{2}}\right)$, the metric is obtained from (2.4) by the replacements $\mathrm{d} s^{2} \rightarrow \widetilde{\mathrm{d} s^{2}}$ and $\overline{\mathrm{d} s^{2}} \rightarrow \widetilde{\mathrm{d} s^{2}}, \boldsymbol{F}$ is given by (2.2), and $\boldsymbol{H}$ by

$$
\boldsymbol{H}=\alpha_{D}^{\prime} Q \boldsymbol{V} \widetilde{\mathrm{d} s^{2}}
$$

Since $\widetilde{\overline{\mathcal{M}}}$ is orientable, $\boldsymbol{H}(2.7)$ is a globally-defined $(D-2)$-form on $\widetilde{\mathcal{M}}$.

It follows from the properties of fundamental groups of quotient spaces 67] that $\left(\mathcal{M}_{g}, \mathrm{~d} s_{g}^{2}\right)=\left(\widetilde{\mathcal{M}}, \widetilde{\mathrm{d} s^{2}}\right) / G$ and $\left(\mathcal{M}, \mathrm{d} s^{2}\right)=\left(\widetilde{\mathcal{M}}, \widetilde{\mathrm{d} s^{2}}\right) / H$, where $G \simeq \pi_{1}\left(\mathcal{M}_{g}\right)$ is a 

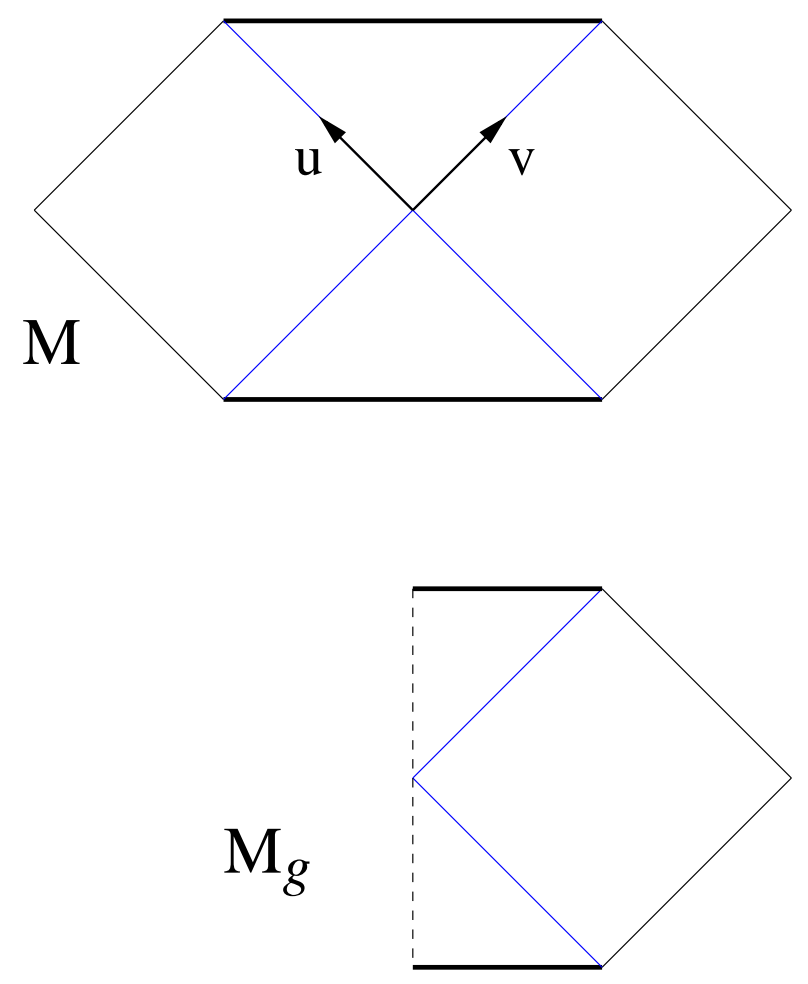

Figure 1: A conformal diagram of the case in which the static regions of $\mathcal{M}$ are asymptotically locally flat and the nonstatic regions terminate at spacelike singularities. The Kruskal null coordinates in (2.4) are chosen to have the range $-\infty<U V<1$, with the infinities at $U V \rightarrow-\infty$ and the singularities at $U V \rightarrow 1$, and the null coordinates $(u, v)$ of the conformal diagram are obtained by $U=\tan (u)$ and $V=\tan (v)$. The conformal diagram of the quotient spacetime $\mathcal{M}_{g}:=\mathcal{M} / \Gamma$ is the (say) right half of that of $\mathcal{M}$, with generic points in the diagram representing a suppressed $\overline{\mathcal{M}}$ but the points on the dashed line on the left representing a suppressed $\overline{\mathcal{M}} / \bar{\Gamma}$.

discrete group of isometries of $\left(\widetilde{\mathcal{M}}, \widetilde{\mathrm{d} s^{2}}\right)$, acting freely and properly discontinuously, $H \simeq \pi_{1}(\mathcal{M}) \simeq \pi_{1}(\overline{\mathcal{M}})$ is a normal subgroup of $G$ and $G / H \simeq \Gamma \simeq \mathbb{Z}_{2}$. By restricting the action of $G$ to the invariant submanifold at $U=0=V$, we obtain from the groups $H \subsetneq G$ the isomorphic groups $\bar{H} \subsetneq \bar{G}$ of isometries of $\left(\widetilde{\overline{\mathcal{M}}}, \widetilde{\mathrm{d} s^{2}}\right)$. It follows that the action of $\bar{G}$ is free and properly discontinuous, $\left(\overline{\mathcal{M}}, \overline{\mathrm{d} s^{2}}\right) / \bar{\Gamma}=\left(\widetilde{\mathcal{M}}, \widetilde{\mathrm{d} s^{2}}\right) / \bar{G}$ and $\left(\overline{\mathcal{M}}, \overline{\mathrm{d} s^{2}}\right)=\left(\widetilde{\overline{\mathcal{M}}}, \widetilde{\mathrm{d} s^{2}}\right) / \bar{H}$.

Let $\bar{G}_{+} \subset \bar{G}$ be the normal subgroup that preserves the orientation on $\widetilde{\mathcal{M}}$, and let $G_{+} \subset G$ be the corresponding subgroup defined by the isomorphism $\bar{G} \simeq G . \bar{G} / \bar{G}_{+}$is then trivial if $\overline{\mathcal{M}} / \bar{\Gamma}$ is orientable and isomorphic to $\mathbb{Z}_{2}$ if $\overline{\mathcal{M}} / \bar{\Gamma}$ is nonorientable. The following proposition shows that three qualitatively different situations arise. 
Proposition 2.1 The subgroups $\bar{H} \subsetneq \bar{G}$ and $\bar{G}_{+} \subset \bar{G}$ can occur in the following three combinations:

(i) $\overline{\mathcal{M}}$ is orientable and $P$ reverses its orientation. Then $\bar{H}=\bar{G}_{+} \subsetneq \bar{G} . \mathcal{M}_{g}$ is orientable.

(ii) $\overline{\mathcal{M}}$ is orientable and $P$ preserves its orientation. Then $\bar{H} \subsetneq \bar{G}_{+}=\bar{G} . \mathcal{M}_{g}$ is nonorientable.

(iii) $\overline{\mathcal{M}}$ is nonorientable. Then $\bar{H} \cap \bar{G}_{+} \subset \bar{G}$ is a normal subgroup and $\bar{G} /\left(\bar{H} \cap \bar{G}_{+}\right)$is canonically isomorphic to $(\bar{G} / \bar{H}) \times\left(\bar{G} / \bar{G}_{+}\right) \simeq \mathbb{Z}_{2} \times \mathbb{Z}_{2}$. $\mathcal{M}_{g}$ is nonorientable.

Proof. Types (i) and (ii) are clear. In Type (iii), the subgroups $\bar{H} \subsetneq \bar{G}$ and $\bar{G}_{+} \subsetneq \bar{G}$ partition $\bar{G}$ into four subsets, at least three of which are nonempty by assumption. Examination of the quotient groups $\bar{G} / \bar{H} \simeq \mathbb{Z}_{2} \simeq \bar{G} / \bar{G}_{+}$shows that all four are nonempty and establishes the isomorphism.

Consider now the gauge field configurations in view of this structure. In the quotient of $\left(\widetilde{\mathcal{M}}, \widetilde{\mathrm{d} s^{2}}\right)$ by $G$, the electric field strength (2.2) needs to be twisted by $G / H$, while the magnetic field strength (2.7) needs to be twisted by $G / G_{+}$, where in each case the nontrivial $\mathbb{Z}_{2}$ element multiplies the form by -1 . The isomorphism $G \simeq \pi_{1}\left(\mathcal{M}_{g}\right)$ shows that this twisting defines the field strength as a section of a bundle over $\mathcal{M}_{g}$. Note that as $\left(\mathcal{M}_{g}, \mathrm{~d} s_{g}^{2}\right)$ has a global foliation by spacelike hypersurfaces, $\pi_{1}\left(\mathcal{M}_{g}\right)$ is isomorphic to the spatial fundamental group of $\mathcal{M}_{g}$.

All the electric and magnetic geons have respectively a nonvanishing electric and magnetic charge at the single asymptopia. This is not forbidden by Stokes' theorem because of the nontriviality of the field strength bundle and/or the role of (non)orientability in Stokes' theorem [64, 65, 66]: the only untwisted field strength occurs for a Type (ii) magnetic geon, which is nonorientable. ${ }^{4}$ Note that the only field strength that is twisted by the (spatial) orientation bundle of $\mathcal{M}_{g}$ occurs in a Type (ii) electric geon.

In the special case $D=4$, in which the electric and magnetic field strengths are both locally two-forms, we can obtain further geons by starting from a linear combination. On the one hand, such constructions are constrained by the requirement that the electric and magnetic parts of the field strength must be sections of the same bundle (so that the linear combination is well-defined). But, on the other hand, we have the opportunity obtain further geons by using the Hodge dual to twist the bundle. Let $\boldsymbol{F}_{0}$ and $\boldsymbol{H}_{0}$ stand for respectively (2.2) and (2.7) on $\left(\widetilde{\mathcal{M}}, \widetilde{\mathrm{d} s^{2}}\right)$. The linear combination

$$
\boldsymbol{F}_{\beta}:=\cos (\beta) \boldsymbol{F}_{0}+\sin (\beta) \boldsymbol{H}_{0}
$$

solves then the two-form Einstein-U(1) equations on $\left(\widetilde{\mathcal{M}}, \widetilde{\mathrm{d} s^{2}}\right)$ for any $\beta \in \mathbb{R}$, and we may understand $\beta$ identified with period $2 \pi$. Fix now the orientation on $\widetilde{\mathcal{M}}$, for

\footnotetext{
${ }^{4}$ It was noted in 64, 65, 66] that an untwisted magnetic field strength on a nonorientable manifold with one asymptopia can have nonvanishing charge. That the $\mathbb{R P}^{3}$ geon does not generalise to accommodate an untwisted electric field was noted in terms of the initial data already in [5].
} 
concreteness but without loss of generality so that $* \boldsymbol{F}_{\beta}=\boldsymbol{F}_{\beta+\pi / 2}$. For the three types in Proposition 2.1] we then obtain solutions as follows:

Type (i). Since $H=G_{+}$, no additional constraints arise from taking the electric and magnetic parts of the field strength to be sections of the same bundle. Thus, $\beta$ is arbitrary and the nontrivial element of $G / H=G / G_{+} \simeq \mathbb{Z}_{2}$ multiplies the field strength by -1 . However, the Hodge dual offers no further inequivalent possibilities.

Type (ii). $\beta=\pi / 4$ or $\beta=5 \pi / 4$ and the nontrivial element of $G / H \simeq \mathbb{Z}_{2}$ acts on the field strength by the Hodge dual. There is no contradiction with the fact that the Hodge dual squares to minus identity, since the nontrivial element of $G / H$ reverses the orientation on $\widetilde{\mathcal{M}}$ and the Hodge dual acquires an additional minus sign on orientation reversal.

Type (iii). $\beta=\pi / 4$ or $\beta=5 \pi / 4$, the nontrivial element of $G / H$ acts on the field strength by the Hodge dual and the nontrivial element of $G / G_{+}$by the inverse of the Hodge dual. As the nontrivial elements of both $G / H$ and $G / G_{+}$reverse the orientation on $\widetilde{\mathcal{M}}$, there is again no contradiction with the Hodge dual squaring to minus identity.

\subsection{Examples: Constant curvature transversal space}

We have seen that classifying geons within the Gibbons-Wiltshire metrics reduces to classifying quotients of the transversal space $\left(\widetilde{\mathcal{M}}, \widetilde{\mathrm{d} s^{2}}\right)$. We now examine these quotients in the special case of constant curvature $\left(\widetilde{\mathcal{M}}, \widetilde{\mathrm{d} s^{2}}\right)$.

\section{$\bar{\lambda}=1:$ Spherical spaces}

When $\bar{\lambda}=1,\left(\widetilde{\mathcal{M}}, \widetilde{\mathrm{d} s^{2}}\right)$ is $(D-2)$-dimensional round sphere, $S^{D-2}$. The quotients are classified in 68 . For any $D$, we can take $\bar{G}$ to be the order two group generated by the antipodal map: This is within our Type (i) for even $D$ and Type (ii) for odd $D$. For even $D$ there are no other possibilities. For odd $D, S^{D-2}$ has infinitely many other quotients satisfying our subgroup assumptions, all of them Type (ii). ${ }^{5}$ The simplest examples arise when $\bar{G}$ is a cyclic group of even order and $\bar{H}$ is the subgroup that consists of even powers of the generator; other examples arise by setting the angular momenta in the rotating geons of subsection 3.2 to zero. Note that the only case in which the spatial topology at infinity is the conventional $S^{D-3}$ is when $\bar{G}$ is generated by the antipodal map.

\footnotetext{
${ }^{5}$ Type (ii) is the only possibility because all quotients of odd-dimensional spheres are orientable. A reader who wishes to forgo the pleasure of verifying this from the full list of quotients in 68. may prefer the following argument. Let $D$ be odd and let $S^{D-2} / L$ be a spherical space, where $L \subset \mathrm{O}(D-1)$ is a subgroup with a free and properly discontinuous action. Suppose $l \in L$ is an element that reverses the orientation of $S^{D-2}$, and let $L_{0} \subset L$ be the subgroup generated by $l$. Then $S^{D-2} / L_{0}$ is a spherical space. If $L_{0}$ has order 2 , it follows from page 218 in 68 that $l$ is the antipodal map, but this is a contradiction since the antipodal map for odd $D$ preserves the orientation. If $L_{0}$ has order greater than 2, it falls into type I of 68. In this case equation (7.4.1) and Theorems 5.5.6 and 5.5.11 in 68. show that all elements in $L_{0}$ preserve the orientation, which is again a contradiction.
} 
When $D=4$ and the form fields vanish, the only possibility is the $\mathbb{R P}^{3}$ geon $[5$, 13 , 14, 15] for $\Lambda=0$ and its asymptotically anti-de Sitter generalisation for $\Lambda<0$.

\section{$\bar{\lambda}=0:$ Flat spaces}

When $\bar{\lambda}=0,\left(\widetilde{\overline{\mathcal{M}}}, \widetilde{\mathrm{d} s^{2}}\right)$ is flat $\mathbb{R}^{D-2}$. Quotients of Types (i), (ii) and (iii) exist for all $D$. For $D=4$, compact examples are obtained when the map $\left(\overline{\mathcal{M}}, \overline{\mathrm{d} s^{2}}\right) \rightarrow\left(\overline{\mathcal{M}}, \overline{\mathrm{d} s^{2}}\right) / \bar{\Gamma}$ is respectively (i) double covering of a Klein bottle by a torus, (ii) double covering of a torus by a torus, and (iii) double covering of a Klein bottle by a Klein bottle. Taking a product with a flat $(D-3)$-torus produces compact examples for $D>4$. Other examples arise by setting the angular momenta in the rotating geons of subsection 3.1 to zero.

\section{$\bar{\lambda}=-1:$ Hyperbolic spaces}

When $\bar{\lambda}=-1,\left(\widetilde{\mathcal{M}}, \widetilde{\mathrm{d} s^{2}}\right)$ is $(D-2)$-dimensional hyperbolic space. Quotients of Types (i), (ii) and (iii) exist for all $D$. For $D>4$, noncompact examples arise by writing the metric in Poincare coordinates,

$$
\widetilde{\mathrm{d} s^{2}}=\frac{\mathrm{d} z^{2}+\mathrm{d} \mathbf{x}^{2}}{z^{2}}
$$

where $z>0$ and $\mathrm{d} \mathbf{x}^{2}$ is the standard flat metric on $\mathbb{R}^{D-3}$, and letting $\bar{G}$ act on $\mathbf{x} \in \mathbb{R}^{D-3}$ as in the $\bar{\lambda}=0$ case. For $D=4$, an example of Type (ii) arises when $\bar{G}$ is generated by a hyperbolic or elliptic Möbius transformation, and an example of Type (i) arises when $\bar{G}$ is generated by a glide-reflection, a hyperbolic Möbius transformation followed by the reflection about the axis of this transformation. An example of Type (iii) arises when $\bar{G}$ is generated by a glide-reflection $A$ and a hyperbolic or elliptic Möbius transformation $B$, with the parameters chosen so that the group acts freely (cf. the pairs of hyperbolic elements analysed in [69]), and $\bar{H}$ is generated by $A$ and $B^{2}$. Compact $D=4$ examples are discussed in 70, 71].

\subsection{Generalisations}

We now consider some generalisations beyond the Gibbons-Wiltshire spacetimes.

The quotient technique generalises immediately to brane-like solutions in which the brane dimensions are sufficiently inert. An example in vacuum with $\Lambda=0$ is the product of Kruskal with any positive definite Ricci-flat space. The brane dimensions may offer new choices for the isometry $P$, and this freedom may in particular be used to make spatially orientable or nonorientable geons as desired. As an example, in the stringy black hole that is the product of the spinless BTZ hole and $S^{3} \times T^{4}$, choosing $P$ to have a suitable nontrivial action on the $T^{4}$ factor gives a spatially orientable geon 22 .

The technique also generalises immediately to form fields of more general rank with a pure $\boldsymbol{F}^{2}$ action. A generalisation to $[\mathrm{U}(1)]^{n}$ form fields is possible and gives new 
opportunities to twist the bundle by permuting the $U(1)$ components. Terms other than $\boldsymbol{F}^{2}$ in a form field action may however bring about new phenomena. For example, a Chern-Simons term, such as that in 11-dimensional supergravity [2], must be a top rank form when the spacetime is orientable but a top rank density when the spacetime is nonorientable. Finally, including dilatonic scalar fields, such as those in [55, 73], is straightforward.

There exist generalisations without an asymptotic region of the kind we have assumed above. One example is the Bertotti-Robinson-type extremal limit of (2.1), in which the spacetime is the product of $(1+1)$-dimensional anti-de Sitter space and a constant multiple of $\left(\overline{\mathcal{M}}, \overline{\mathrm{d} s^{2}}\right)$. The horizon is then a Killing horizon on the anti-de Sitter space, and the quotient analysis proceeds as in subsection 2.2. In the limit of vanishing curvature, these spacetimes reduce to geon-like versions of Rindler space [18].

Another example is de Sitter space. The geon-like quotient yields a version in which the spacelike hypersurfaces in the global foliation are not $S^{D-1}$ but $S^{D-1} / \mathbb{Z}_{2} \simeq \mathbb{R} \mathbb{P}^{D-1}$ 74, 75, 76].

Yet another example occurs when $\Lambda>0$ and the conformal diagram is as for the nondegenerate Schwarzschild-de Sitter solution, with a pattern of black hole and cosmological horizons repeating infinitely in the horizontal direction [77]. It is possible to take a $\mathbb{Z}_{2}$ quotient using one of the horizons, either a black hole horizon or a cosmological one. The conformal diagram becomes then bounded from (say) the left and infinite to the right. However, it is also possible to take a quotient under a group generated by two involutive isometries, each using a different horizon, so that the conformal diagram becomes bounded both from the left and from the right [76]. In this case the fixed timelike hypersurfaces of the two isometries can be arbitrarily boosted with respect to each other (cf. the discussion in two-dimensional dilaton gravity context in [78, Figure 14). These quotients might offer an arena for probing dS/CFT correspondence [79, 80].

Finally, the higher-dimensional BTZ hole [48, 81, 82, 83, 84, resembles a geon in that the exterior region is connected. It is however possible to take a $\mathbb{Z}_{2}$ quotient of this hole, with the effect that the topology at infinity changes and the isometry group becomes smaller. These properties were investigated in the AdS/CFT context in [85].

\section{Angular momentum}

In this section we construct rotating geons. We consider first angular momenta on a torus and then angular momenta on a (non-round) sphere.

\section{$3.1 \quad$ Torus}

Let $\left(\mathcal{N}, \mathrm{d} s^{2}\right)$ be the planar vacuum Gibbons-Wiltshire hole with $\Lambda<0$. The metric reads

$$
\mathrm{d} s^{2}=-f \mathrm{~d} U \mathrm{~d} V+r^{2} \mathrm{~d} \ell^{2}
$$


where $\mathrm{d} \ell^{2}$ is the standard flat positive definite metric on $\mathbb{R}^{D-2}$ and $f$ and the range of the Kruskal coordinates $(U, V)$ are as in (2.4) with $\Lambda<0=\bar{\lambda}$. Let $1 \leq d \leq D-2$, let $\left\{\boldsymbol{\xi}^{(\alpha)} \mid \alpha=1, \ldots, d\right\}$ be a linearly independent set of translational Killing vectors on $\left(\mathbb{R}^{D-2}, \mathrm{~d} \ell^{2}\right)$, and let

$$
\boldsymbol{\eta}^{(\alpha)}:=\boldsymbol{\xi}^{(\alpha)}+a^{(\alpha)}\left(V \partial_{V}-U \partial_{U}\right)
$$

where $a^{(\alpha)}$ are real-valued constants, assumed so small in absolute value that $\boldsymbol{\eta}^{(\alpha)} \cdot \boldsymbol{\eta}^{(\alpha)} \rightarrow$ $\infty$ as $r \rightarrow \infty$ for each $\alpha .\left\{\boldsymbol{\eta}^{(\alpha)}\right\}$ is a set of commuting Killing vectors on $\left(\mathcal{N}, \mathrm{d} s^{2}\right)$. The group $H_{0}$ generated by the exponential maps of these Killing vectors acts freely and properly discontinuously, and the quotient $\left(\mathcal{N}, \mathrm{d} s^{2}\right) / H_{0}=:\left(\mathcal{N}_{0}, \mathrm{~d} s_{0}^{2}\right)$ is an eternal black hole with spatial topology $T^{d} \times \mathbb{R}^{D-2-d}$.

When all the $a$ 's vanish, we are in the situation covered in section 2 Assume from now on that at least one of the $a$ 's is nonvanishing. $\left(\mathcal{N}_{0}, \mathrm{~d} s_{0}^{2}\right)$ is then a spinning eternal black hole and the $a$ 's have an interpretation in terms of angular momenta 42, 44, 86.

In taking a geon quotient of $\left(\mathcal{N}_{0}, \mathrm{~d} s_{0}^{2}\right)$ we face a new difficulty in that the map $(U, V) \mapsto(V, U)$ on $\left(\mathcal{N}, \mathrm{d} s^{2}\right)$ reverses the signs of the $a$ 's. The angular momenta at the two infinities, defined with respect to the global time orientation on $\left(\mathcal{N}_{0}, \mathrm{~d} s_{0}^{2}\right)$, therefore have opposite signs. There do exist time-orientation preserving isometries between the two exterior regions, but in general such an isometry needs to invert all the spatial dimensions, which implies that the isometry does not extend into an involutive fixedpoint free isometry of $\left(\mathcal{N}_{0}, \mathrm{~d} s_{0}^{2}\right)$.

However, in certain special configurations a geon quotient exists. We give two examples.

First, suppose that $d \geq 2, \boldsymbol{\xi}^{(1)}$ is orthogonal to all others $\boldsymbol{\xi}^{\prime}$ s and $a^{(1)}=0$. We introduce on $\left(\mathbb{R}^{D-2}, \mathrm{~d} \ell^{2}\right)$ the adapted coordinates $\boldsymbol{y}=\left(y^{1}, \boldsymbol{y}_{\perp}\right) \in \mathbb{R} \times \mathbb{R}^{D-3} \simeq \mathbb{R}^{D-2}$, in which $\boldsymbol{\xi}^{(1)}=b \partial_{y^{1}}$. Consider on $\left(\mathcal{N}, \mathrm{d} s^{2}\right)$ the isometry

$$
J_{1}:\left(U, V, y^{1}, \boldsymbol{y}_{\perp}\right) \mapsto\left(V, U, y^{1}+(b / 2),-\boldsymbol{y}_{\perp}\right)
$$

which preserves $\boldsymbol{\eta}^{(1)}$ and reverses the signs of all other $\boldsymbol{\eta}$ 's. Let $G_{0}$ be the group generated by $H_{0}$ and $J_{1}$. $G_{0}$ acts on $\left(\mathcal{N}, \mathrm{d} s^{2}\right)$ freely and properly discontinuosly, $H_{0} \subset G_{0}$ is a normal subgroup with $G_{0} / H_{0} \simeq \mathbb{Z}_{2}$ and $\left(\mathcal{N}, \mathrm{d} s^{2}\right) / G_{0} \simeq\left(\mathcal{N}_{0}, \mathrm{~d} s_{0}^{2}\right) / \mathbb{Z}_{2}$ is a geon. There can be up to $D-3$ independent angular momentum parameters.

Second, suppose $D \geq 5, d \geq 3, \boldsymbol{\xi}^{(1)}$ and $\boldsymbol{\xi}^{(2)}$ have equal magnitude and are orthogonal to all other $\boldsymbol{\xi}$ 's and the only nonvanishing $a$ 's are $a^{(1)}=-a^{(2)}$. Introduce on $\left(\mathbb{R}^{D-2}, \mathrm{~d} \ell^{2}\right)$ the adapted coordinates $\boldsymbol{y}=\left(\left(y^{1}, y^{2}\right), \boldsymbol{y}_{\perp}\right) \in \mathbb{R}^{2} \times \mathbb{R}^{D-4} \simeq \mathbb{R}^{D-2}$, in which $\boldsymbol{\xi}^{(1)}=b \partial_{y^{1}}$ and $\boldsymbol{\xi}^{(2)}=b \partial_{y^{2}}$. (Note that $\boldsymbol{\xi}^{(1)}$ and $\boldsymbol{\xi}^{(2)}$ need not be orthogonal.) Consider on $\left(\mathcal{N}, \mathrm{d} s^{2}\right)$ the isometry

$$
J_{2}:\left(U, V, y^{1}, y^{2}, \boldsymbol{y}_{\perp}\right) \mapsto\left(V, U, y^{2}, y^{1}, \exp \left(\frac{1}{2} \boldsymbol{\xi}^{(3)}\right) \boldsymbol{y}_{\perp}\right)
$$

which interchanges $\boldsymbol{\eta}^{(1)}$ and $\boldsymbol{\eta}^{(2)}$ and preserves all other $\boldsymbol{\eta}$ 's. The quotient of $\left(\mathcal{N}, \mathrm{d} s^{2}\right)$ by the group generated by $H_{0}$ and $J_{2}$ is then a $\mathbb{Z}_{2}$ geon quotient of $\left(\mathcal{N}_{0}, \mathrm{~d} s_{0}^{2}\right)$.

For some block-diagonal configurations there exist $\mathbb{Z}_{2}$ quotients in which the involutive isometry acts as with $J_{1}$ in some blocks and as with $J_{2}$ in others. Also, for some 
configurations further rotating geons may be constructed by a quotient that has order higher than 2 , using maps that generalise $J_{2}$ to permute an even number of dimensions. We shall not attempt to enumerate all the possibilities here.

Dilatonic fields and antisymmetric tensor fields can be included as with the nonrotating geons.

\subsection{Sphere}

We consider in $D \geq 4$ dimensions the rotating Myers-Perry(-AdS) black holes [28, 29, 30, 31, 32, which solve the vacuum Einstein equations with a vanishing (respectively negative) cosmological constant. We assume positive mass and a nondegenerate horizon. We use the Kruskal coordinates that were explicitly given for a vanishing cosmological constant in 28] and whose construction for a negative cosmological constant is similar.

In the Kruskal coordinates of [28], a left-right reflection in the conformal diagram reverses the signs of all the angular momenta. An isometry $K$ that involves such a reflection thus needs to include some compensating operation in the $D-2$ dimensions suppressed in the diagram. In order that $K$ can be used in the quotient, the group generated by $K$ needs to act freely and properly discontinuously. If the quotient is further required to be continuously deformable to the nonrotating case, no such $K$ exists for even $D$ or for $D=7$. For even $D$, the reason is that in the nonrotating limit $\bar{G}$ must be the order two group generated by the antipodal map, as discussed in subsection 2.3. as the nontrivial element of $G$ then leaves all the (nonrotating) rotation planes invariant, this element cannot be the limit of an isometry that permutes (rotating) rotation planes. For $D=7$, the reason is that in the nonrotating limit $\bar{G}$ must be either the order two group generated by the antipodal map or one of the higher order isometry groups of the round 5-sphere listed on p. 225 in 68. The only nontrivial action of $G$ on the three commuting (nonrotating) rotation planes is then a cyclic permutation, and this cannot be the limit of an action on a rotating spacetime since a cyclic permutation of an odd number of angular momenta is equivalent to an overall sign change only when all the angular momenta in fact vanish.

When $D$ is odd and not equal to 7 , rotating geons exist. We give an example.

First, suppose $D=4 p+1$ where $p$ is a positive integer. We define the null Kruskal coordinates $(U, V)$ by $U:=v-u$ and $V:=v+u$, where $u$ and $v$ are respectively the spacelike and timelike Kruskal coordinates of [28. We denote the first $p$ rotation angles by $\boldsymbol{\theta}=\left(\theta_{1}, \ldots, \theta_{p}\right)$, the associated direction cosines by $\boldsymbol{\mu}=\left(\mu_{1}, \ldots, \mu_{p}\right)$, the remaining $p$ rotation angles by $\boldsymbol{\varphi}=\left(\varphi_{1}, \ldots, \varphi_{p}\right)$ and the associated direction cosines by $\boldsymbol{\nu}=$ $\left(\nu_{1}, \ldots, \nu_{p}\right)$. Recall that all the direction cosines are non-negative and satisfy $\boldsymbol{\mu}^{2}+\boldsymbol{\nu}^{2}=1$, each rotation angle is periodic with period $2 \pi$, and that coordinate singularities only occur when the vanishing of a direction cosine makes the corresponding rotation angle ambiguous.

Assume now the angular momentum in each $\theta_{i}$ to be the negative of the angular momentum in the corresponding $\varphi_{i}$. Let $q$ and $r_{i}, 1 \leq i \leq p$, be positive integers such 
that $\operatorname{gcd}\left(r_{i}, 2 q\right)=1$ for all $i$. Consider the map

$$
K_{1}:(U, V, \boldsymbol{\mu}, \boldsymbol{\nu}, \boldsymbol{\theta}, \boldsymbol{\varphi}) \mapsto(V, U, \boldsymbol{\nu}, \boldsymbol{\mu}, \boldsymbol{\varphi}, \boldsymbol{\theta}+(\pi / q) \boldsymbol{r})
$$

where $\boldsymbol{r}:=\left(r_{1}, \ldots, r_{p}\right)$. In words, $K_{1}$ effects a left-right reflection in the conformal diagram, interchanges all the paired rotation planes, and rotates in the last $p$ rotation planes by $\pi r_{i} / q . K_{1}$ generates an isometry group of order $4 q$, this group acts freely and properly discontinuously, and the quotient is a geon. The geon is asymptotically locally flat, the spatial infinity being an order $2 q$ quotient of $S^{4 p-1}$ and reducing to $\mathbb{R} \mathbb{P}^{4 p-1}$ for $q=1$. The geon can be written as the $\mathbb{Z}_{2}$ quotient of the two-exterior hole that is the quotient of the original spacetime under the order $q$ isometry group generated by $K_{1}^{2}$.

Second, suppose $D=4 p+7$ where $p$ is a positive integer. We denote the first $2 p$ rotation angles and direction cosines as above, the remaining three rotation angles by $\left(\psi_{1}, \psi_{2}, \psi_{3}\right)$ and their direction cosines by $\left(\rho_{1}, \rho_{2}, \rho_{3}\right)$. The direction cosines are nonnegative and satisfy $\boldsymbol{\mu}^{2}+\boldsymbol{\nu}^{2}+\rho_{1}^{2}+\rho_{2}^{2}+\rho_{3}^{2}=1$, and the periodicities and coordinate singularities are as above. Assume again the angular momentum in each $\theta_{i}$ to be the negative of the angular momentum in the corresponding $\varphi_{i}$, and assume in addition the angular momentum in each $\psi_{i}$ to vanish. Let $q$ and $s$ be positive integers satisfying $4 q=9 s$, let $r_{i}$ be as above, and let $t$ be a positive integer satisfying $\operatorname{gcd}(t, 3 s)=1$. Consider the map

$$
\begin{aligned}
& K_{2}:\left(U, V, \boldsymbol{\mu}, \boldsymbol{\nu}, \rho_{1}, \rho_{2}, \rho_{3}, \boldsymbol{\theta}, \boldsymbol{\varphi}, \psi_{1}, \psi_{2}, \psi_{3}\right) \mapsto \\
& \quad\left(V, U, \boldsymbol{\nu}, \boldsymbol{\mu}, \rho_{2}, \rho_{3}, \rho_{1}, \boldsymbol{\varphi}, \boldsymbol{\theta}+(\pi / q) \boldsymbol{r}, \psi_{2}, \psi_{3}, \psi_{1}+2 \pi t /(3 s)\right) .
\end{aligned}
$$

In words, $K_{2}$ acts in the conformal diagram and the first $2 p$ rotation planes as $K_{1}$, permutes the last three (non-rotating) rotation planes cyclically and rotates in one of them by $2 \pi t /(3 s) . K_{2}$ generates an isometry group of order $4 q=9 s$, this group acts freely and properly discontinuously, and the quotient is a geon. Spatial infinity is an order $2 q$ quotient of $S^{4 p+5}$, the lowest possible value of $q$ now being 9 .

In both examples the geon has the $p$ rotational Killing vectors $\partial_{\theta_{i}}+\partial_{\varphi_{i}}, i=1, \ldots, p$, and in the second example there is an additional rotational Killing vector $\partial_{\psi_{1}}+\partial_{\psi_{2}}+$ $\partial_{\psi_{3}}$. The angular momenta with respect to all of these Killing vectors vanish. The $p$ independent nonvanishing angular momenta are defined with respect to the Killing line fields $\pm\left(\partial_{\theta_{i}}-\partial_{\varphi_{i}}\right), i=1, \ldots, p$, which can be defined as vector fields in a neighbourhood of infinity but only as line fields on the whole geon. That a rotational Killing line field can have nonvanishing angular momentum on a manifold with one asymptopia was noted in the four-dimensional setting in 66].

Further examples arise by generalising the isometries in each $\left(\theta_{i}, \varphi_{i}\right)$ pair and in the $\left(\psi_{1}, \psi_{2}, \psi_{3}\right)$ triple in non-cyclic ways that can be read off from the list of block-diagonal isometries of the round 3-sphere and the round 5-sphere on pages 224-225 in [68], and also by using isometries that cyclically permute larger numbers of angles. For example, when there are $2 p$ nonvanishing angular momenta such that the first $p$ are equal and the remaining $p$ have the opposite sign, we can arrange the isometries to permute the 
$2 p$ rotation planes cyclically. We shall not attempt to enumerate all the possibilities. We suspect however that the quotients shown above have the largest possible number of unequal angular momenta.

Inclusion of dilatonic fields and antisymmetric tensor fields can in principle be discussed as in section 2, although few such solutions are at present explicitly known 87, 88, 89, 90, 91, 92, 93.

\section{Einstein-SU(2) geon}

Since the initial discovery of spherically symmetric asymptotically flat Einstein-SU(2) black holes [33, 34, a large variety of Einstein-Yang-Mills black holes have been found. A fairly recent survey is given in 94. The nonabelian gauge freedom makes investigation of geon quotients substantially more complicated than in the abelian case, especally as the known solutions tend to be given in gauges in which the spacetime symmetries are not manifest. We address here only one example, the four-dimensional spherically symmetric Einstein-SU(2) hole in which the cosmological constant may be negative or vanishing [33, 34, 35, 36, 37, 38, 39. We show that this hole has a geon variant with a trivial gauge bundle.

We follow the notation of [37]. The exterior metric is written in Schwarzschild-like coordinates as

$$
\mathrm{d} s^{2}=-\frac{H}{p^{2}} \mathrm{~d} t^{2}+\frac{\mathrm{d} r^{2}}{H}+r^{2} \mathrm{~d} \Omega^{2}
$$

where $H$ and $p$ are functions of $r, \mathrm{~d} \Omega^{2}=\mathrm{d} \theta^{2}+\sin ^{2} \theta \mathrm{d} \varphi^{2}$ is the metric on the unit two-sphere and $(\theta, \varphi)$ are the usual angle coordinates. There is a nondegenerate horizon at $r=r_{h}>0$, the exterior is at $r_{h}<r<\infty$, and the metric functions have the near-horizon expansions

$$
\begin{aligned}
& H=\sum_{k=1}^{\infty} h_{k}\left(r-r_{h}\right)^{k}, \\
& p=1+\sum_{k=1}^{\infty} p_{k}\left(r-r_{h}\right)^{k},
\end{aligned}
$$

where $h_{1}>0$. Transforming the gauge field configuration from the singular gauge used in [37. to a regular gauge, by the matrix (9) in 37] with $\Omega=\pi / 2$, we obtain the gauge potential

$$
\boldsymbol{A}^{(0)}=\frac{1}{2 e}\left[(\vec{n} \cdot \vec{\tau}) u \mathrm{~d} t+(w-1) \epsilon_{i j k} \tau^{i} n^{j} \mathrm{~d} n^{k}\right]
$$

where $\tau^{i}$ are the Pauli matrices as in [37], $\vec{n}:=(\sin \theta \cos \varphi, \sin \theta \sin \varphi, \cos \theta)$, and $u$ and 
$w$ are functions of $r$ with the near-horizon expansions

$$
\begin{gathered}
u=\sum_{k=1}^{\infty} u_{k}\left(r-r_{h}\right)^{k}, \\
w=\sum_{k=0}^{\infty} w_{k}\left(r-r_{h}\right)^{k} .
\end{gathered}
$$

We define the Kruskal coordinates in the exterior by $U=-\exp \left[-\frac{1}{2} h_{1}(t-\rho)\right]$ and $V=\exp \left[\frac{1}{2} h_{1}(t+\rho)\right]$, where $\mathrm{d} \rho / \mathrm{d} r=p / H$ and we choose the additive constant in $\rho$ so that $\rho=h_{1}^{-1} \ln \left[\left(r-r_{h}\right)\right]+\mathcal{O}\left(\left(r-r_{0}\right)\right)$. The horizon is at $U V \rightarrow 0, r$ is a function of $U V$ with the Taylor expansion $r=r_{h}-U V+\mathcal{O}\left((U V)^{2}\right)$, and the metric takes the form

$$
\mathrm{d} s^{2}=-f \mathrm{~d} U \mathrm{~d} V+r^{2} \mathrm{~d} \Omega^{2}
$$

where $f$ is a function of $U V$ with the Taylor expansion $f=4 h_{1}^{-1}+\mathcal{O}(U V)$. The gauge potential (4.3) takes the form

$$
\boldsymbol{A}^{(0)}=\frac{1}{2 e}\left[(\vec{n} \cdot \vec{\tau}) g(V \mathrm{~d} U-U \mathrm{~d} V)+(w-1) \epsilon_{i j k} \tau^{i} n^{j} \mathrm{~d} n^{k}\right]
$$

where $g$ is a function of $U V$ with the Taylor expansion $g=u_{1} h_{1}^{-1}+\mathcal{O}(U V)$. The solution given by (4.5) and (4.6) is clearly extendible across $U V=0$ into a two-exterior black hole spacetime $\left(\mathcal{M}, \mathrm{d} s^{2}\right)$ in the standard fashion. As $\boldsymbol{A}^{(0)}$ (4.6) then becomes a globally-defined $\mathfrak{s u}(2)$-valued 1 -form on $\mathcal{M}$, the gauge bundle over $\mathcal{M}$ is trivial.

Now, the only involutive freely-acting isometry on the two-sphere is the antipodal map $P:(\theta, \varphi) \mapsto(\pi-\theta, \varphi+\pi)$, or $\vec{n} \mapsto-\vec{n}$. With this $P$, the isometry $J$ (2.6) plainly leaves $\boldsymbol{A}^{(0)}$ (4.6) invariant. The quotient of $\left(\mathcal{M}, \mathrm{d} s^{2}\right)$ by $\left\{\operatorname{Id}_{\mathcal{M}}, J\right\}$ is therefore a geon with a trivial gauge bundle. The ranges of the magnetic and electric charges in the geon are exactly as in the two-exterior hole.

The difference from the spherically symmetric U(1) geons, in which both the electric and magnetic field strengths needed to be twisted, arises from the nontrivial $\mathfrak{s u}(2)-$ valuedness of the gauge field. It can be verified that the 'electric' and 'magnetic' parts of the field strength computed from $\boldsymbol{A}^{(0)}$ (4.6) are both in a hedgehog-like $\mathfrak{s u}(2)$ configuration over the two-sphere.

\section{Conclusions and discussion}

In this paper we have constructed new families of geon-type black holes in $D \geq 4$ dimensions for Einstein's theory coupled to gauge fields, by taking $\mathbb{Z}_{2}$ quotients of eternal black hole solutions that have a nondegenerate Killing horizon. In the static vacuum case, the existence of the quotient was equivalent to the existence of suitable isometries on the spatial sections, and antisymmetric tensor fields with a pure $\boldsymbol{F}^{2}$ action could then always be included by taking the field strength to be a section of a suitable, and 
in most cases nontrivial, bundle. With angular momentum, we constructed rotating geons as quotients of the Myers-Perry(-AdS) solution when $D$ is odd and not equal to 7 . These rotating geons are asymptotically flat (anti-de Sitter, respectively), but the spatial infinity is a non-trivial quotient of the usual sphere. For other $D$ we showed that rotating geon quotients of the Myers-Perry(-AdS) solution, if they exist at all, cannot be continuously deformed to the static case. With a negative cosmological constant, we constructed geons with angular momenta on a torus at the infinity. Finally, we showed that the $D=4$ spherically symmetric $\mathrm{SU}(2)$ black hole admits a geon quotient with a trivial gauge bundle.

In the Gibbons-Wiltshire geons of section 2, we analysed the abelian gauge field configuration in terms of the field strength, rather than in terms of the gauge potential. In the electric geons and in the $D=4$ magnetic geons, the gauge field can be interpreted as a connection in a principal $\mathrm{O}(2)$ bundle. For the electric geons and for the magnetic $D=4$ geons with $\bar{\lambda} \leq 0$, this follows by first interpreting the gauge field on the universal covering space as a connection in the trivial $\mathrm{O}(2)$ bundle and then using the disconnected component of $\mathrm{O}(2)$ to twist the bundle on taking the quotient. For the $D=4$ magnetic geon with $\bar{\lambda}=1$, a similar argument is not available since the $\mathrm{U}(1) \simeq \mathrm{SO}(2)$ bundle on the universal covering space is already nontrivial, but the existence of the $\mathrm{O}(2)$ bundle can be verified directly. ${ }^{6}$ For the $D>4$ magnetic geons, the gauge potential issue would need to be addressed in terms of gerbes [95].

All our solutions have a high degree of symmetry. For the $D=4$ solutions with an abelian gauge field, this symmetry is dictated by the uniqueness theorems for stationary black holes [96]. The uniqueness theorems are known not to have a straightforward generalisation to $D>4$ [97, 98, and there are suggestions that the uniqueness violation could be severe 99. With a nonabelian gauge field, black holes with less symmetry are known to occur even in the static $D=4$ context [100, 101, 102]. One would like to understand to what extent our geon quotients generalise to black holes with less symmetry. One would also like to understand at a more general level what kind of hair a geon may have. For example, despite the similarities between the spherically symmetric $\mathrm{SU}(2)$ black hole and the spherically symmetric skyrmion black hole of [103, 104, 105, the skyrmion field configuration is not invariant under the isometry that yielded the $\mathrm{SU}(2)$ geon in section 4 . What is the situation for, say, the spherically symmetric $\mathrm{SU}(n)$ black holes with $n>2$ [106]?

From the viewpoint of string theory, it would be of interest to explore geon versions of black holes that are solutions to supergravity limits of M-theory. String theoretic dualities have provided a microscopic state-counting explanation for the entropy of certain families of such black holes [107]: can D-brane states corresponding to geons be identified and distinguished from those corresponding to other black holes? If yes, do such states successfully account for the physical entropy of the geon?

\footnotetext{
${ }^{6}$ This is essentially the observation that the $\mathrm{SO}(2)$ monopole on $S^{2}$ with an even monopole number can be quotiented into an $\mathrm{O}(2)$ monopole on $\mathbb{R}^{2}$.
} 


\section{Acknowledgements}

We thank John Barrett, John Friedman, Nico Giulini, Eli Hawkins, Viqar Husain, HansPeter Künzle, Todd Oliynyk, Simon Ross, David Wiltshire, Elizabeth Winstanley and Jacek Wiśniewski for helpful discussions. J. L. was supported in part by the Engineering and Physical Sciences Research Council. R. B. M. was supported in part by the Natural Sciences and Engineering Research Council of Canada. D. M. was supported in part by NSF grant PHY03-54978 and by funds from the University of California. 


\section{References}

[1] J. A. Wheeler, "Geons", Phys. Rev. 97, 511 (1955).

[2] D. R. Brill and J. A. Wheeler, "Interaction of neutrinos and gravitational fields", Rev. Mod. Phys. 29, 465 (1957).

[3] F. J. Ernst, Jr., "Variational calculations in geon theory", Phys. Rev. 105, 1662 (1957).

[4] F. J. Ernst, Jr., "Linear and toroidal geons", Phys. Rev. 105, 1665 (1957).

[5] C. W. Misner and J. A. Wheeler, "Classical physics as geometry: Gravitation, electromagnetism, unquantized charge, and mass as properties of curved empty space" Ann. Phys. (NY) 2, 525 (1957). Reprinted in: J. A. Wheeler, Geometrodynamics (Academic, New York, 1962).

[6] D. R. Brill and J. B. Hartle, "Method of the self-consistent field in general relativity and its application to the gravitational geon", Phys. Rev. 135, B271 (1964).

[7] M. A. Melvin, "Pure magnetic and electric geons", Phys. Lett. 8, 65 (1963).

[8] M. A. Melvin, "Dynamics of cylindrical electromagnetic universes", Phys. Rev. 139, B225 (1965).

[9] K. S. Thorne, "Energy of infinitely long, cylindrically symmetric systems in general relativity", Phys. Rev. 138, B251 (1965).

[10] K. S. Thorne, "Absolute Stability of Melvin's Magnetic Universe", Phys. Rev. 139, B244 (1965).

[11] C. Gundlach, "Critical phenomena in gravitational collapse", Phys. Reports 376, 339 (2003). 〈arXiv: gr-qc/0210101〉

[12] R. D. Sorkin, "Introduction to topological geons", in: Topological properties and global structure of space-time, Proceedings of the NATO Advanced Study Institute on on Topological Properties and Global Structure of Space-Time, Erice, Italy, May 12-22, 1985, edited by P. G. Bergmann and V. De Sabbata (Plenum, 1986), pp. 249-270.

[13] J. L. Friedman, K. Schleich and D. M. Witt, "Topological censorship", Phys. Rev. Lett. 71, 1486 (1993); Erratum, Phys. Rev. Lett. 75, 1872 (1995). $\langle$ arXiv:gr-qc/9305017〉

[14] D. Giulini, "3-manifolds in canonical quantum gravity", Ph.D. Thesis, University of Cambridge (1990). 
[15] D. Giulini, "Two-body interaction energies in classical general relativity", in: Relativistic Astrophysics and Cosmology, Proceedings of the Tenth Seminar, Potsdam, October 21-26 1991, edited by S. Gottlöber, J. P. Mücket and V. Müller (World Scientific, Singapore, 1992), pp. 333-338.

[16] G. Szekeres, "On the singularities of a Riemannian manifold", Publ. Mat. Debrecen 7, 285 (1960).

[17] A. Chamblin and G. W. Gibbons, "Nucleating black holes via nonorientable instantons", Phys. Rev. D 55, 2177 (1997). 〈arXiv:gr-qc/9607079〉

[18] J. Louko and D. Marolf, "Inextendible Schwarzschild black hole with a single exterior: How thermal is the Hawking radiation?" Phys. Rev. D 58, 024007 (1998). 〈arXiv:gr-qc/9802068〉

[19] J. Louko and B. F. Whiting, "Hamiltonian thermodynamics of the Schwarzschild black hole", Phys. Rev. D 51, 5583 (1995). 〈arXiv:gr-qc/9411017〉

[20] J. L. Friedman, J. Louko and S. N. Winters-Hilt, "Reduced phase space formalism for spherically symmetric geometry with a massive dust shell", Phys. Rev. D 56, 7674 (1997). 〈arXiv:gr-qc/9706051〉

[21] P. Langlois, "Hawking radiation for Dirac spinors on the $\mathbb{R P}^{3}$ geon", Phys. Rev. D 70, 104008 (2004). 〈arXiv:gr-qc/0403011〉

[22] J. Louko and D. Marolf, "Single-exterior black holes and the AdS-CFT conjecture", Phys. Rev. D 59, 066002 (1999). 〈arXiv:hep-th/9808081〉

[23] J. Louko, D. Marolf and S. F. Ross, "Geodesic propagators and black hole holography", Phys. Rev. D 62, 044041 (2000). 〈arXiv:hep-th/0002111〉

[24] J. M. Maldacena, "Eternal black holes in anti-de Sitter", JHEP 0304, 021 (2003). $\langle\operatorname{arXiv:hep-th/0106112\rangle }$

[25] S. Åminneborg, I. Bengtsson and S. Holst, "A spinning anti-de Sitter wormhole", Class. Quantum Grav. 16, 363 (1999). 〈arXiv:gr-qc/9805028〉

[26] D. Brill, "Black holes and wormholes in 2+1 dimensions", in: Mathematical and Quantum Aspects of Relativity and Cosmology, Proceedings of 2nd Samos Meeting on Cosmology, Geometry and Relativity Karlovasi, Greece, 31 August - 4 September 1998 (Lecture Notes in Physics, Vol. 537), edited by S. Cotsakis and G. W. Gibbons (Springer, Berlin, 2000), pp. 143-179. 〈arXiv:gr-qc/9904083〉

[27] D. Brill, “(2+1)-dimensional black holes with momentum and angular momentum", Annalen Phys. (Leipzig) 9, 217 (2000). 〈arXiv:gr-qc/9912079〉 
[28] R. C. Myers and M. J. Perry, "Black holes in higher-dimensional spacetimes", Ann. Phys. (N.Y.) 172, 304 (1986).

[29] S. W. Hawking, C. J. Hunter and M. M. Taylor-Robinson, "Rotation and the AdS/CFT correspondence", Phys. Rev. D 59, 064005 (1999). $\langle$ arXiv:hep-th/9811056

[30] G. W. Gibbons, H. Lü, D. N. Page and C. N. Pope, "Rotating black holes in higher dimensions with a cosmological constant", Phys. Rev. Lett. 93, 171102 (2004). 〈arXiv:hep-th/0409155〉

[31] G. W. Gibbons, H. Lü, D. N. Page and C. N. Pope, "The general Kerr-de Sitter metrics in all dimensions", J. Geom. Phys. 53, 49 (2005). $\langle$ arXiv:hep-th/0404008〉

[32] G. W. Gibbons, M. J. Perry and C. N. Pope, "The first law of thermodynamics for Kerr-Anti-de Sitter black holes". 〈arXiv:hep-th/0408217〉

[33] P. Bizon, "Colored black holes", Phys. Rev. Lett. 64, 2844 (1990).

[34] H. P. Künzle and A. K. M. Masood-ul-Alam, "Spherically symmetric static SU(2) Einstein-Yang-Mills fields", J. Math. Phys. 31, 928 (1990).

[35] E. Winstanley, "Existence of stable hairy black holes in SU(2) Einstein-Yang-Mills theory with a negative cosmological constant", Class. Quantum Grav. 16, 1963 (1999). 〈arXiv:gr-qc/9812064〉

[36] J. Bjoraker and Y. Hosotani, "Stable monopole and dyon solutions in the EinsteinYang-Mills theory in asymptotically anti-de Sitter space", Phys. Rev. Lett. 84, 1853 (2000). 〈arXiv:gr-qc/9906091〉

[37] J. Bjoraker and Y. Hosotani, "Monopoles, dyons and black holes in the fourdimensional Einstein-Yang-Mills theory", Phys. Rev. D 62, 043513 (2000). $\langle$ arXiv:hep-th/0002098〉

[38] E. Winstanley and O. Sarbach, "On the linear stability of solitons and hairy black holes with a negative cosmological constant: The even parity sector", Class. Quantum Grav. 19, 689 (2002). 〈arXiv:gr-qc/0111039〉

[39] E. Winstanley and O. Sarbach, "On the linear stability of solitons and hairy black holes with a negative cosmological constant: The odd parity sector", Class. Quantum Grav. 18, 2125 (2001). 〈arXiv:gr-qc/0102033〉

[40] G. W. Gibbons and D. L. Wiltshire, "Space-time as a membrane in higher dimensions", Nucl. Phys. B287, 717 (1987). 〈arXiv:hep-th/0109093〉 
[41] J. P. S. Lemos, "Two-dimensional black holes and planar general relativity", Class. Quantum Grav. 12, 1081 (1995).〈arXiv:gr-qc/9407024〉

[42] J. P. S. Lemos, "Cylindrical black hole in general relativity", Phys. Lett. B353, 46 (1995). 〈arXiv:gr-qc/9404041〉

[43] C. Huang and C. Liang, "A torus-like black hole", Phys. Lett. A201, 27 (1995).

[44] J. P. S. Lemos and V. T. Zanchin, "Rotating charged black string and threedimensional black holes", Phys. Rev. D 54, 3840 (1996). 〈arXiv:hep-th/9511188〉

[45] R. B. Mann, "Pair production of topological anti-de Sitter black holes", Class. Quantum Grav. 14, L109 (1997). 〈arXiv:gr-qc/9607071〉

[46] R. Cai and Y. Zhang, "Black plane solutions in four dimensional spacetimes", Phys. Rev. D 54, 4891 (1996). 〈arXiv:gr-qc/9609065〉

[47] W. L. Smith and R. B. Mann, "Formation of topological black holes from gravitational collapse", Phys. Rev. D 56, 4942 (1977). 〈arXiv:gr-qc/9703007〉

[48] M. Bañados, "Constant curvature black holes", Phys. Rev. D 57, 1068 (1998). $\langle$ arXiv:gr-qc/9703040

[49] L. Vanzo, "Black holes with unusual topology", Phys. Rev. D 56, 6475 (1997). $\langle$ arXiv:gr-qc/9705004〉

[50] R. Mann, "Black holes of negative mass", Class. Quantum Grav. 14, 2927 (1997). $\langle$ arXiv:gr-qc/9705007〉

[51] D. R. Brill, J. Louko and P. Peldán, "Thermodynamics of $(3+1)$-dimensional black holes with toroidal or higher genus horizons", Phys. Rev. D 56, 3600 (1997). $\langle$ arXiv:gr-qc/9705012〉

[52] D. Birmingham, "Topological black holes in anti-de Sitter space", Class. Quantum Grav. 16, 1197 (1999). 〈arXiv:hep-th/9808032〉

[53] R. Emparan, C. V. Johnson and R. C. Myers, "Surface terms as counterterms in the AdS/CFT correspondence", Phys. Rev. D 60, 104001 (1999). $\langle$ arXiv:hep-th/9903238〉

[54] R. Emparan, "AdS/CFT duals of topological black holes and the entropy of zero energy states", JHEP 9906, 036 (1999). 〈arXiv:hep-th/9906040〉

[55] G. W. Gibbons and K. Maeda, "Black holes and membranes in higher-dimensional theories with dilaton fields", Nucl. Phys. B298, 741 (1988).

[56] R. Bott and L. W. Tu, Differential Forms in Algebraic Topology (Springer, New York, 1982). 
[57] R. M. Wald, Quantum Field Theory in Curved Spacetime and Black Hole Thermodynamics (The University of Chicago Press, Chicago, 1994).

[58] S. W. Hawking and G. F. R. Ellis, The Large Scale Structure of Space-Time (Cambridge University Press, Cambridge, 1973).

[59] K. Lake, "Reissner-Nordström-de Sitter metric, the third law, and cosmic censorship", Phys. Rev. D 19, 421 (1979).

[60] T. Klösch and T. Strobl, "Classical and quantum gravity in $1+1$ dimensions. II: The universal coverings", Class. Quantum Grav. 13, 2395 (1996). $\langle$ arXiv:gr-qc/9511081〉

[61] L. Fidkowski, V. Hubeny, M. Kleban and S. Shenker, "The black hole singularity in AdS/CFT", JHEP 0402, 014 (2004). 〈arXiv:hep-th/0306170〉

[62] G. Galloway, K. Schleich and D. M. Witt, "Topological censorship and higher genus black holes", Phys. Rev. D 60, 104039 (1999). 〈arXiv:gr-qc/9902061〉

[63] G. Galloway, K. Schleich, D. M. Witt and E. Woolgar, "The AdS/CFT correspondence conjecture and topological censorship", Phys. Lett. B505, 255 (2001). $\langle\operatorname{arXiv:hep-th/9912119\rangle }$

[64] R. Sorkin, "On the relation between charge and topology", J. Phys. A 10, 717 (1977).

[65] R. Sorkin, "The quantum electromagnetic field in multiply connected space", J. Phys. A 12, 403 (1979).

[66] J. L. Friedman and S. Mayer, "Vacuum handles carrying angular momentum; electrovac handles carrying net charge", J. Math. Phys. 23, 109 (1982).

[67] A. Hatcher, Algebraic Topology (Cambridge University Press, Cambridge, 2002), Proposition 1.40 and Exercise 1.3.24.

[68] J. A. Wolf, Spaces of Constant Curvature, 5th edition (Publish or Perish, Wilmington, 1984).

[69] G. T. Horowitz and D. Marolf, "A new approach to string cosmology", JHEP 9807, 014 (1998). 〈arXiv:hep-th/9805207〉

[70] M. Schiffer and D. C. Spencer, Functionals of finite Riemann surfaces (Princeton University Press, Princeton, New Jersey, 1954).

[71] N. L. Alling and N. Greenleaf, Foundations of the theory of Klein surfaces, Lecture Notes in Mathematics Vol. 219 (Springer, Berlin, 1971). 
[72] M. J. Duff, B. E. W. Nilsson and C. N. Pope, "Kaluza-Klein supergravity", Phys. Reports 130, 1 (1986).

[73] K. C. K. Chan, J. Horne and R. B. Mann, "Charged Dilaton black holes with unusual asymptotics", Nucl. Phys. B447, 441 (1995).〈arXiv:gr-qc/9502042〉

[74] J. Louko and K. Schleich, "The exponential law: Monopole detectors, Bogolubov transformations, and the thermal nature of the Euclidean vacuum in $\mathbb{R P}^{3}$ de Sitter spacetime", Class. Quantum Grav. 16, 2005 (1999). 〈arXiv:gr-qc/9812056〉

[75] K. Schleich and D. M. Witt, "The generalized Hartle-Hawking initial state: Quantum field theory on Einstein conifolds", Phys. Rev. D 60, 064013 (1999). $\langle$ arXiv:gr-qc/9903062〉

[76] B. McInnes, "de Sitter and Schwarzschild-de Sitter according to Schwarzschild and de Sitter", JHEP 0309, 009 (2003). 〈arXiv:hep-th/0308022〉

[77] G. W. Gibbons and S. W. Hawking, "Cosmological event horizons, thermodynamics, and particle creation", Phys. Rev. D 15, 2738 (1977).

[78] T. Klösch and T. Strobl, "Classical and quantum gravity in 1+1 dimensions. III: Solutions with arbitrary topology", Class. Quantum Grav. 14, 1689 (1997). $\langle$ arXiv:hep-th/9607226〉

[79] E. Witten, "Quantum gravity in de Sitter space", in: New Fields and Strings in Subnuclear Physics, edited by A. Zichichi (Singapore, World Scientific, 2003) [Int. J. Mod. Phys. A 18, Supplement (2003)]. 〈arXiv:hep-th/0106109〉

[80] A. Strominger, "The dS/CFT correspondence", JHEP 0110, 034 (2001). $\langle\operatorname{arXiv:hep-th/0106113\rangle }$

[81] S. Åminneborg, I. Bengtsson, S. Holst and P. Peldán, "Making anti-de Sitter black holes", Class. Quantum Grav. 13, 2707 (1996).〈arXiv:gr-qc/9604005〉

[82] S. Holst and P. Peldán, "Black holes and causal structure in anti-de Sitter isometric spacetimes", Class. Quantum Grav. 14, 3433 (1997). 〈arXiv:gr-qc/9705067〉

[83] J. D. E. Creighton and R. B. Mann, "Entropy of constant curvature black holes in general relativity", Phys. Rev. D 58, 024013 (1998). 〈arXiv:gr-qc/9710042〉

[84] M. Bañados, A. Gomberoff and C. Martínez, "Anti-de Sitter space and black holes", Class. Quantum Grav. 15, 3575 (1998). 〈arXiv:hep-th/9805087〉

[85] J. Louko and J. Wiśniewski, "Einstein black holes, free scalars and AdS/CFT correspondence", Phys. Rev. D 70084024 (2004). 〈arXiv:hep-th/0406140〉

[86] A. M. Awad, "Higher-dimensional charged rotating solutions in (A)dS spacetimes", Class. Quantum Grav. 20, 2827 (2003). 〈arXiv:hep-th/0209238〉 
[87] M. Cvetic and D. Youm, "Near BPS saturated rotating electrically charged black holes as string states", Nucl. Phys. B477, 449 (1996). 〈arXiv:hep-th/9605051〉

[88] P. M. Llatas, "Electrically charged black holes for the heterotic string compactified on a $(10-D)$ torus", Phys. Lett. A397, 63 (1997). 〈arXiv:hep-th/9605058〉

[89] M. Cvetič, H. Lü and C. N. Pope, "Charged Kerr-de Sitter black holes in five dimensions", Phys. Lett. B598, 273 (2004). 〈arXiv:hep-th/0406196〉

[90] M. Cvetič, H. Lü and C. N. Pope, "Charged rotating black holes in five dimensional $\mathrm{U}(1)^{3}$ gauged $\mathcal{N}=2$ supergravity", Phys. Rev. D 70081502 (2004). $\langle$ arXiv:hep-th/0407058〉.

[91] O. Madden and S. F. Ross, "On uniqueness of charged Kerr-AdS black holes in five dimensions", Class. Quantum Grav. 22515 (2005). 〈arXiv:hep-th/0409188〉.

[92] Z.-W. Chong, M. Cvetič, H. Lü and C. N. Pope, "Charged rotating black holes in four-dimensional gauged and ungauged supergravities", 〈arXiv:hep-th/0411045〉

[93] Z.-W. Chong, M. Cvetič, H. Lü and C. N. Pope, "Non-extremal charged rotating black holes in seven-dimensional gauged supergravity", 〈arXiv: hep-th/0412094〉

[94] M. S. Volkov and D. V. Gal'tsov, "Gravitating non-abelian solitons and black holes with Yang-Mills fields", Phys. Rept. 319, 1 (1999). 〈arXiv:hep-th/9810070〉

[95] M Mackaay and R. Picken, "Holonomy and parallel transport for abelian gerbes", Adv. Math. 170, 287 (2002). 〈arXiv:math.dg/0007053〉

[96] M. Heusler, Black Hole Uniqueness Theorems (Cambridge University Press, Cambridge, 1996).

[97] R. Emparan and H. S. Reall, "A rotating black ring solution in five dimensions", Phys. Rev. Lett. 88, 101101 (2002). 〈arXiv:hep-th/0110260〉

[98] H. Elvang, R. Emparan, D. Mateos and H. S. Reall, "A supersymmetric black ring", Phys. Rev. Lett. 93211302 (2004). 〈arXiv:arXiv:hep-th/0407065〉.

[99] H. S. Reall, "Higher dimensional black holes and supersymmetry", Phys. Rev. D 68, 024024 (2003). 〈arXiv:hep-th/0211290〉

[100] B. Kleihaus and J. Kunz, "Static black hole solutions with axial symmetry", Phys. Rev. Lett. 79, 1595 (1997). 〈arXiv:gr-qc/9704060〉

[101] B. Kleihaus and J. Kunz, "Static axially symmetric Einstein-Yang-Mills dilaton solutions. II. Black hole solutions', Phys. Rev. D 57, 6138 (1998). $\langle$ arXiv:gr-qc/9712086〉 
[102] E. Radu and E. Winstanley, "Static axially symmetric solutions of Einstein-YangMills equations with a negative cosmological constant: Black hole solutions", Phys. Rev. D 70, 084023 (2004). 〈arXiv:hep-th/0407248〉

[103] H. Luckock and I. Moss, "Black holes have skyrmion hair", Phys. Lett. B176, 341 (1986).

[104] H. Luckock, "Black hole skyrmions", in: String theory, quantum cosmology and quantum gravity: Proceedings of the Paris-Meudon Colloquium 22-26 September 1986 (World Scientific, Singapore, 1987), pp. 454-464.

[105] S. Droz, M. Heusler and N. Straumann, "New black hole solutions with hair", Phys. Lett. B268, 371 (1991).

[106] H. P. Künzle, "Analysis of the static spherically symmetric $\mathrm{SU}(N)$ Einstein YangMills equations", Commun. Math. Phys. 162, 371 (1994).

[107] A. W. Peet, "TASI lectures on black holes in string theory," in: Strings, Branes, and Gravity: TASI 99: Boulder, Colorado, 31 May - 25 June 1999, edited by J. Harvey, S. Kachru, and E. Silverstein (World Scientific, Singapore, 2001), pp. 353-433. 〈arXiv:hep-th/0008241〉 